TRANSACTIONS OF THE

AMERICAN MATHEMATICAL SOCIETY

Volume 362, Number 9, September 2010, Pages 4557-4568

S 0002-9947(10)04808-7

Article electronically published on April 27, 2010

\title{
ON THE CHARACTERIZATION OF ALGEBRAICALLY INTEGRABLE PLANE FOLIATIONS
}

\author{
C. GALINDO AND F. MONSERRAT
}

\begin{abstract}
We give a characterization theorem for non-degenerate plane foliations of degree different from 1 having a rational first integral. Moreover, we prove that the degree $r$ of a non-degenerate foliation as above provides the minimum number, $r+1$, of points in the projective plane through which pass infinitely many algebraic leaves of the foliation.
\end{abstract}

\section{INTRODUCTION}

At the end of the 19th century, Darboux [9, Poincaré [20, 21, 22, Painlevé [19] and Autonne [1] studied the problem that, nowadays, can be stated as follows: to characterize those algebraic foliations on the projective plane over the field of complex numbers (plane foliations, in the sequel) which have a rational first integral. Most recent contributions related to that problem are focused on solving the socalled Poincaré problem, which consists of bounding the degrees of the irreducible algebraic leaves of a foliation in terms of numerical data related to it [7, 5, 17, 11. From this bound, one can try to get a rational first integral through an algebraic computation.

In this note, we only consider non-degenerate plane foliations $\mathcal{F}$. When $\mathcal{F}$ has a rational first integral $R$, the singular points of $\mathcal{F}$ are, on the one hand, the indeterminacies of the rational map $f_{R}: \mathbb{P}^{2} \cdots \rightarrow \mathbb{P}^{1}$ which $R$ defines $(\operatorname{Ind}(R))$ and, on the other hand, the singular points of the fibers of this rational map $(\operatorname{Sing}(R))$. In the case considered in this paper, non-degenerate foliations, $\operatorname{Ind}(R)(\operatorname{Sing}(R)$, respectively) coincides with the set of non-reduced (reduced, respectively) singularities of $\mathcal{F}$, denoted by $N \operatorname{Red}(\mathcal{F})(\operatorname{Red}(\mathcal{F})$, respectively).

In Proposition 3.6, for a plane foliation $\mathcal{F}$ of degree different from 1 , we consider the set of rational functions $R$ such that $\operatorname{Ind}(R)=N \operatorname{Red}(\mathcal{F})$ and $\operatorname{Sing}(R)=$ $\operatorname{Red}(\mathcal{F})$ and characterize when an element of that set is a rational first integral of $\mathcal{F}$ in terms of local conditions on the singularities of the closures of the fibers of $f_{R}$. These conditions only depend on the analytic type of the mentioned singularities. An important ingredient of the proof is the fact, proved by Campillo and Olivares

Received by the editors November 10, 2006 and, in revised form, April 18, 2008.

2010 Mathematics Subject Classification. Primary 32S65.

The first author was supported by the Spain Ministry of Education MTM2007-64704 and Bancaixa P1-1B2009-03.

The second author was supported by the Spain Ministry of Education MTM2007-64704 and Bancaixa P1-1A2005-08. 
in [6], that foliations of degree different from one are determined by the scheme associated with the indeterminacies of the polarity map of the foliation.

If we know Seidenberg's resolution of $\mathcal{F}$ [24, the ideas used to prove the above result can be strengthened to get our main result, Theorem 3.7, which is a characterization theorem for foliations of degree different from 1 having a rational first integral. The characterization conditions are two numerical ones (related to the eigenvalues of the non-reduced singularities) and other conditions that have to do with a certain sheaf on the surface obtained after Seidenberg's resolution process. Note that when $\mathcal{F}$ is of degree one and all its singularities are non-degenerate, with rational quotients of eigenvalues, then it always has a rational first integral.

In the previous results, an important object is the set $N \operatorname{Red}(\mathcal{F})$. For this reason, we end this paper by proving in Theorem 3.13 that, under the assumption that $\mathcal{F}$ is algebraically integrable, the cardinality of $N \operatorname{Red}(\mathcal{F})$ is greater than the degree of $\mathcal{F}$. Notice that this means that the degree of a non-degenerate foliation, $r$, provides the minimum number, $r+1$, of points in $\mathbb{P}^{2}$ through which pass infinitely many leaves of the foliation.

\section{Plane foliations AND Algebraic integrability}

Along this note, $\mathbb{P}^{2}$ will denote the complex projective plane, $\mathcal{O}_{\mathbb{P}^{2}}\left(\mathcal{O}_{\mathbb{P}^{2}}^{a n}\right.$, respectively) its structural sheaf as an algebraic variety over $\mathbb{C}$ (its sheaf of holomorphic functions, respectively). A plane foliation or a foliation on $\mathbb{P}^{2}, \mathcal{F}$, will be an (algebraic) foliation (with singularities and singular set of codimension two) of degree $r(r \geq 0)$ on $\mathbb{P}^{2}$. That is, $\mathcal{F}$ is a non-trivial map of vector bundles $\mathcal{F}: H^{\otimes(-r+1)} \rightarrow T \mathbb{P}^{2}$ whose cokernel is torsion-free, where $T \mathbb{P}^{2}$ and $H$ are the corresponding bundles to the tangent and hyperplane sheaves on $\mathbb{P}^{2}$ and $H^{\otimes n}$ means the $n$-fold tensor product of $H$ if $n>0$, the $(-n)$-fold tensor product of $H^{\vee}$ if $n<0$ and the trivial line bundle if $n=0$.

A plane foliation $\mathcal{F}$ provides a tangent direction (or, equivalently, a projective line) to all points in $\mathbb{P}^{2}$ but finitely many called singularities of $\mathcal{F}$, a set denoted by $\operatorname{Sing}(\mathcal{F})$. This allows us to define the so-called polarity map $\phi: \mathbb{P}^{2} \cdots \rightarrow\left(\mathbb{P}^{2}\right)^{\vee}$, that is, the rational map which sends each point $p \in \mathbb{P}^{2} \backslash \operatorname{Sing}(\mathcal{F})$ to that point in $\left(\mathbb{P}^{2}\right)^{\vee}$ corresponding to the projective line that $\mathcal{F}$ associates with $p$. The schemetheoretic fibers $\phi^{*} E$ of the lines $E$ in $\left(\mathbb{P}^{2}\right)^{\vee}$ are degree $-(r+1)$ curves on $\mathbb{P}^{2}$ and so, since lines in $\left(\mathbb{P}^{2}\right)^{\vee}$ can be identified with points in $\mathbb{P}^{2}$, we can assign to each point $p \in \mathbb{P}^{2}$ a curve of degree $r+1$ called the polar of $p$ with respect to $\mathcal{F}$. The indeterminacy ideal $\mathcal{J}$ of the polarity map gives a subscheme of $\mathbb{P}^{2}$, named the singular subscheme of $\mathcal{F}$, which determines the foliation [6.

$\mathcal{F}$, in analytic terms, can be defined, up to multiplication by a non-zero complex number, by a reduced homogeneous vector field (up to a suitable multiple of the radial vector field) or, equivalently, by a reduced homogeneous 1-form, $\Omega \equiv A d X+$ $B d Y+C d Z,(X: Y: Z)$ being projective coordinates on $\mathbb{P}^{2}$, such that $A, B, C$ are homogeneous polynomials of degree $(r+1)$ in $X, Y, Z$ without common factors and satisfying the Euler condition $X A+Y B+Z C=0$. This last condition is what allows us to recover $\Omega$ from local data, which will be considered over the corresponding affine charts of $\mathbb{P}^{2}$. For example, $\Omega$ over the chart $Z \neq 0$ will be expressed by $\omega=a(x, y) d x+b(x, y) d y$, where $x=X / Z, y=Y / Z, a(x, y)=A(X, Y, 1), b(x, y)=$ $B(X, Y, 1)$. As a consequence, a point $p$ in the above chart will be a singularity of $\mathcal{F}$ if, and only if, $a(p)=b(p)=0$. Recall that the colength of the stalk $\mathcal{J}_{p}$ of the 
above-defined indeterminacy ideal (which is the ideal $(a, b) \mathcal{O}_{\mathbb{P}^{2}, p}$ in the coordinates $\{x, y\})$ in the local ring $\mathcal{O}_{\mathbb{P}^{2}, p}, \mu_{p}(\mathcal{F})$, is called the Milnor number of $\mathcal{F}$ at the point $p$.

For fixed homogeneous coordinates on $\mathbb{P}^{2}$, a rational function $R$ of $\mathbb{P}^{2}$ is defined by the quotient of two homogeneous polynomials in these coordinates without common components and with the same degree, $F$ and $G$. Associated with $R$, we consider the linear pencil $\mathcal{P}(R)$ consisting of the projective curves with equations $\alpha F+\beta G=0$, where $(\alpha, \beta)$ runs over $\mathbb{C}^{2} \backslash\{0\}$. The curves in $\mathcal{P}(R)$ are the topological closures of the fibers of the rational map $\mathbb{P}^{2} \cdots \rightarrow \mathbb{P}^{1}$ that $R$ defines. The base locus of $\mathcal{P}(R)$ is the set of indeterminacies of this map. As we have said, it will be denoted by $\operatorname{Ind}(R)$. Also, we shall denote by $\operatorname{Sing}(R)$ the set of singular points of the curves of the pencil $\mathcal{P}(R)$ which are not in $\operatorname{Ind}(R)$.

By definition, a foliation $\mathcal{F}$ has a rational (or meromorphic) first integral if there exists a rational function $S$ of $\mathbb{P}^{2}$ such that $d S \wedge \Omega=0$. In this case, there exists a rational function $R=F / G$ such that all the rational first integrals of $\mathcal{F}$ have the form $\frac{P(F, G)}{Q(F, G)}$, where $P, Q$ are homogeneous polynomials of the same degree; such a function $R$ will be called a primitive rational first integral and it satisfies that its associated pencil of plane curves $\mathcal{P}(R)$ is irreducible (which means that it has irreducible general elements). Moreover, the leaves of $\mathcal{F}$ are the algebraic curves whose irreducible components are components of some curve of the mentioned pencil.

We only consider foliations $\mathcal{F}$, which we shall call non-degenerate ones, such that the Milnor numbers of all its singularities equal one. For each singularity $p$ of $\mathcal{F}$, the pair $(\delta, \rho)$ of eigenvalues of the linear part of any local vector field defining $\mathcal{F}$ at $p$ is named the (pair of) eigenvalues associated with $p$. Notice that $\delta \neq 0 \neq \rho$. We are interested in algebraic integrability; so we assume that $\delta$ and $\rho$ are integers and that $\operatorname{gcd}(\delta, \rho)=1$, after dividing by its greatest common divisor if it is necessary. When $\delta / \rho$ is not positive, then we shall say that $\mathcal{F}$ has a reduced singularity at $p$. We shall denote by $\operatorname{Red}(\mathcal{F})(N \operatorname{Red}(\mathcal{F})$, respectively) the set of reduced (non-reduced, respectively) singularities.

A well-known fact, which we shall use systematically throughout the paper, is that if $\mathcal{F}$ is a non-degenerate foliation with rational first integral $R$, then $\operatorname{Ind}(R)=$ $N \operatorname{Red}(\mathcal{F})$ and $\operatorname{Sing}(R)=\operatorname{Red}(\mathcal{F})$.

\section{Characterization of Foliations With a RAtional First integral}

In this section, we study the problem of characterizing when a non-degenerate plane foliation has a rational first integral. First of all, we recall some definitions that we shall use in the sequel.

Definition 3.1. A polynomial $P \in \mathbb{C}[x, y]$ is weighted-homogeneous with weights $\left(w_{1}, w_{2}\right)$, where $w_{1}, w_{2}$ are fixed rational numbers, if it can be expressed as a linear combination of monomials $x^{\alpha} y^{\beta}$ for which $\frac{\alpha}{w_{1}}+\frac{\beta}{w_{2}}=1$.

Definition 3.2. A plane curve singularity is quasi-homogeneous if it is analytically equivalent to a singularity whose local equation is defined by a weightedhomogeneous polynomial.

Let $\mathbb{Z}_{+}$stand for the set of positive integers. Given $a, b, k \in \mathbb{Z}_{+}$such that $\operatorname{gcd}(a, b)=1$, we shall denote by $S(a, b, k)$ the topological singularity type of a plane curve singularity with equation $x^{k a}+y^{k b}=0$, where $\{x, y\}$ are local coordinates. 
We shall also consider the following set of topological singularity types:

$$
\mathcal{S}=\left\{S(a, b, k) \mid a, b, k \in \mathbb{Z}_{+} \text {and } \operatorname{gcd}(a, b)=1\right\} .
$$

The topology of a quasi-homogeneous singularity determines the weights of all quasi-homogeneous polynomials defining the singularity [25. Therefore, the quasihomogeneous singularities with singularity type $S(a, b, k)$ correspond to the weights $(k a, k b)$.

Definition 3.3. We shall say that a plane curve singularity is nodal if its local equations have the form $g_{1}^{n} g_{2}^{m}=0$, where $m, n$ are positive integers and $g_{1}, g_{2}$ define analytically irreducible regular germs which are transversal.

Definition 3.4. Let $\mathcal{F}$ be a non-degenerate foliation on $\mathbb{P}^{2}$ and $p$ a non-reduced singular point of $\mathcal{F}$ with associated eigenvalues $(\delta, \rho) . \mathcal{F}$ is said to be linearizable at $p$ if there exist analytical coordinates $\{u, v\}$ at $p$ such that $\mathcal{F}_{p}$ is defined by the differential form $\rho v d u-\delta u d v$.

Although the results of the next lemma are well known for the specialists, we include its proof for the reader's convenience.

Lemma 3.5. Let $\mathcal{F}$ be a non-degenerate foliation on $\mathbb{P}^{2}$ admitting a rational first integral $R$. Then,

(a) $\mathcal{F}$ is linearizable at every non-reduced singularity.

(b) A point $p \in \mathbb{P}^{2}$ is a reduced singularity of $\mathcal{F}$ if, and only if, it is not a base point of $\mathcal{P}(R)$ and the unique curve $H$ of $\mathcal{P}(R)$ passing through $p$ has a nodal singularity at this point.

Proof. For non-resonant singularities, (a) follows from Poincaré's linearization theorem (see, for instance, 4 ) and otherwise, bearing in mind that the singularity is non-reduced, from the Poincaré-Dulac normal form theorem (see [4] and [3, pages 16 and 17]).

To prove (b), assume that $p$ is a reduced singularity. Set $h:=u \prod_{i=1}^{s} h_{i}^{e_{i}}=0$, a local equation of the germ at $p$ of $H$, where $u$ is a unit and $h_{i}=0$ an analytically irreducible germ for $i=1,2, \ldots, s$. Then, $d\left(u^{-1} h\right)=w \prod_{i=1}^{s} h_{i}^{e_{i}-1}$, where $w=$ $a d x+b d y$ with $a, b \in \mathcal{O}_{\mathbb{P}^{2}, p}^{a n}$ and $\operatorname{gcd}(a, b)=1,\{x, y\}$ being local coordinates (see [13, Section 2.4]). Therefore, the multiplicity of $\mathcal{F}$ at $p$ (i.e., the minimum of the $m_{p}$-adic orders of $a$ and $b, m_{p}$ being the maximal ideal of $\left.\mathcal{O}_{\mathbb{P}^{2}, p}^{a n}\right)$ is $\nu=-1+\sum_{i=1}^{s} \nu\left(h_{i}\right)$, where $\nu\left(h_{i}\right)$ denotes the multiplicity of the germ $h_{i}$. Since $\nu=1$, one has that $\sum_{i=1}^{s} \nu\left(h_{i}\right)=2$ and, in particular, either $s=1$ or $s=2$.

If $s=1$, then $h$ is $u\left(x^{2}+f\right)^{e_{1}}$ for some local coordinates $\{x, y\}, f \in(x, y)^{3}$ and $u$ a unit. This shows a local expression of $\mathcal{F}$ at $p$, which, clearly, cannot hold since $\mathcal{F}$ is non-degenerate. If $s=2$, either $h=0$ defines a nodal singularity, or $h=u\left(x+f_{1}\right)^{e_{1}}\left(x+f_{2}\right)^{e_{2}}$ with $f_{1}, f_{2} \in(x, y)^{2}$, which again contradicts the fact that $\mathcal{F}$ is non-degenerate.

The converse is clear since, in certain coordinates $\{x, y\}$, a local equation for $H$ is $h=0$, with $h=(x+f)^{e_{1}}(y+g)^{e_{2}}$ and $f, g \in(x, y)^{2} \subseteq \mathcal{O}_{\mathbb{P}^{2}, p}^{a n}$, and hence, $p$ is a reduced singularity of $\mathcal{F}$.

Recall that if $C$ is a curve, $p$ a point in $C, \eta: \bar{C} \rightarrow C$ the normalization map and $\gamma$ the class map, that is, the composition on the canonical sheaf $\gamma: \Omega_{C}^{1} \rightarrow$ $\eta_{*} \Omega \frac{1}{C} \rightarrow \omega_{C}$, the Milnor (Tjurina, respectively) number of $C$ at $p$ is $\mu(C, p):=$ 
$l\left(\operatorname{Cok}(\gamma \circ d)_{p}\right)\left(\tau(C, p):=l\left(\operatorname{Ext}_{\mathcal{O}_{C, p}}^{1}\left(\Omega_{C, p}^{1}, \mathcal{O}_{C, p}\right)\right)\right.$, respectively), where $d: \mathcal{O}_{C} \rightarrow \Omega_{C}^{1}$ is the universal derivation and $l$ the length.

Proposition 3.6. Let $\mathcal{F}$ be a non-degenerate plane algebraic foliation of degree different from 1 and let $R$ be a rational function such that $\operatorname{Ind}(R)=N \operatorname{Red}(\mathcal{F})$ and $\operatorname{Sing}(R)=\operatorname{Red}(\mathcal{F})$. Then, $R$ is a rational first integral of $\mathcal{F}$ if and only if the following conditions are satisfied:

1. The singularity at every point in $\operatorname{Sing}(R)$ of the curve of $\mathcal{P}(R)$ passing through it is nodal.

2. There exist two curves of the pencil $\mathcal{P}(R)$ (say, with equations $H_{1}=0$ and $\left.H_{2}=0\right)$ such that, for each $p \in \operatorname{Ind}(R)$, the germs at $p$ of the curves with equations $H_{1}=0$ and $H_{2}=0$ are equisingular and the one of $H_{1} H_{2}=0$ satisfies the following properties:

(a) it is a reduced germ whose topological singularity type belongs to $\mathcal{S}$,

(b) its associated Milnor and Tjurina numbers coincide.

Proof. Assume that $R$ is a rational first integral for $\mathcal{F}$. Condition 1 is satisfied by Part (b) of Lemma 3.5 .

In order to prove 2, pick two distinct general elements (with equations $H_{i}=0$, $i=1,2$ ) of the pencil $\mathcal{P}(R))$ such that the local equation at each point $p \in \operatorname{Ind}(R)$ of the curve $C$ defined by $H_{1} H_{2}=0$ is reduced. Consider a point $p \in \operatorname{Ind}(R)$. By Part (a) of Lemma 3.5, there exist local coordinates $u, v \in \mathcal{O}_{\mathbb{P}^{2}, p}^{a n}$ such that the foliation $\mathcal{F}$ is defined locally at $p$ by the differential 1 -form $\rho v d u-\delta u d v$, where $(\delta, \rho)$ is the pair of eigenvalues associated with $p$. If $h_{1}\left(h_{2}\right.$, respectively) in $\mathcal{O}_{\mathbb{P}^{2}, p}^{a n}$ defines the germ provided by $H_{1}\left(H_{2}\right.$, respectively), since $H_{1}=0$ and $H_{2}=0$ are general elements of $\mathcal{P}(R)$ one has that $h_{1}$ and $h_{2}$ give equisingular reduced germs (and, obviously, without common factors). Moreover, since $\frac{u^{\rho}}{v^{\delta}}$ is a primitive local rational first integral of the germ of foliation $\mathcal{F}_{p}$, there exist a unit $z$ and homogeneous polynomials in two variables $P$ and $Q$ of the same degree and without common factors such that $h_{1}=z P\left(u^{\rho}, v^{\delta}\right)$ and $h_{2}=z Q\left(u^{\rho}, v^{\delta}\right)$ (see, for instance, [13, Section 2.9]). $z^{-2} h_{1} h_{2}$ factorizes into a product of polynomials of the type $L\left(u^{\rho}, v^{\delta}\right), L$ being a homogeneous polynomial of degree 1 . Hence, it is clear that Condition 2(a) is satisfied. Finally, notice that $h_{1} h_{2}$ defines a quasi-homogeneous singularity and, then, Condition 2(b) follows from the second "Satz" in [23, page 123].

Conversely, consider the foliation $\mathcal{H}$ provided by the derivation of the rational function $R$. Taking into account the hypotheses of the statement, the equality $\operatorname{Sing}(\mathcal{H})=\operatorname{Sing}(\mathcal{F})$ clearly holds. Next, we shall prove the equality $\mathcal{F}=\mathcal{H}$ and, hence, the result. By applying [6, Th. 3.5], it is enough to show that $\mathcal{H}$ is a non-degenerate foliation.

Let $H_{1}$ and $H_{2}$ be as in Condition 2 and take an arbitrary point $p \in \operatorname{Ind}(R)$. Consider the curve $C$ of equation $H_{1} H_{2}=0$. By Condition 2(b) and the second "Satz" in [23, page 123], $C$ has a quasi-homogeneous singularity at $p$. Therefore, there exist local analytic coordinates $\{u, v\}$ and a quasi-homogeneous polynomial $P$ such that $P(u, v)=0$ defines this singularity. Moreover, applying Condition 2(a), its singularity type belongs to $\mathcal{S}$ and there exist three positive integers $\rho, \delta$ and $k$ such that $\operatorname{gcd}(\rho, \delta)=1$ and $(k \delta, k \rho)$ is the pair of weights corresponding to $P(u, v)$. It is straightforward to see that $P(u, v)=Q\left(u^{\rho}, v^{\delta}\right)$, where $Q$ is a homogeneous polynomial in two variables of degree $k$. Therefore, $P(u, v)=\prod_{i=1}^{k} L_{i}\left(u^{\rho}, v^{\delta}\right), L_{i}$ 
being distinct homogeneous polynomials of degree 1 . Now, $k$ is even and $P(u, v)=$ $Q_{1}\left(u^{\rho}, v^{\delta}\right) Q_{2}\left(u^{\rho}, v^{\delta}\right)$, where $Q_{1}$ and $Q_{2}$ are homogeneous polynomials of degree $k / 2$ and $Q_{1}\left(u^{\rho}, v^{\delta}\right)=0\left(Q_{2}\left(u^{\rho}, v^{\delta}\right)=0\right.$, respectively) is an equation of the germ at $p$ of the curve $H_{1}=0\left(H_{2}=0\right.$, respectively) in the local coordinates $u$ and $v$ (notice that these facts are true because the mentioned germs are equisingular). Taking into account that the derivation of $Q_{1}\left(u^{\rho}, v^{\delta}\right) / Q_{2}\left(u^{\rho}, v^{\delta}\right)$ defines the same local foliation as the germ $\mathcal{H}_{p}$, we deduce that $u^{\rho} / v^{\delta}$ is a primitive local first integral of $\mathcal{H}_{p}$ and, therefore, $\mu_{p}(\mathcal{H})=1$. Finally, Condition 1 ensures that $\mu_{p}(\mathcal{H})=1$ for all $p \in \operatorname{Sing}(R)$, which concludes the proof.

Let $\mathcal{F}$ be a non-degenerate plane foliation. By Seidenberg's resolution process 24, there exists a sequence of point blow-ups whose composition, $\pi: Y \rightarrow \mathbb{P}^{2}$, satisfies that the foliation on $Y \pi^{*}(\mathcal{F})$ has only reduced singularities (see [3, pages 12 and 13]). Such a composition morphism is called a minimal resolution of $\mathcal{F}$ if it is minimal with respect to the number of involved blow-ups. We shall denote by $\pi_{\mathcal{F}}: X_{\mathcal{F}} \rightarrow \mathbb{P}^{2}$ a minimal resolution of singularities of $\mathcal{F}$ and by $\mathcal{C}_{\mathcal{F}}$ the set of centers of the blow-ups that are involved in it (notice that this set is, essentially, unique). $\mathcal{C}_{\mathcal{F}}$ is a disjoint union $\bigcup \mathcal{C}_{\mathcal{F}, p}, p$ running over $N \operatorname{Red}(\mathcal{F})$, where $\mathcal{C}_{\mathcal{F}, p}:=\left\{q \in \mathcal{C}_{\mathcal{F}} \mid q \geq p\right\}$ and $\geq$ is the partial ordering on $\mathcal{C}_{\mathcal{F}}$ given by $q \geq r$ if and only if $q$ is infinitely near to $r$ [8,3.3].

Now we shall define a family of sheaves on $X_{\mathcal{F}}$ associated with this minimal resolution. For each $p \in N \operatorname{Red}(\mathcal{F})$, we distinguish two cases:

- $\mathcal{F}$ is linearizable at $p$. Then there exist local coordinates $u, v \in \mathcal{O}_{\mathbb{P}^{2}, p}^{a n}$ such that $\mathcal{F}_{p}$ is defined by the differential 1 -form $\rho v d u-\delta u d v,(\delta, \rho)$ being the pair of eigenvalues associated with $p$. Let $J_{p}$ be the ideal of $\mathcal{O}_{\mathbb{P}^{2}, p}^{a n}$ generated by $u^{\rho}$ and $v^{\delta}$. Elementary computations show that the infinitely near points needed to eliminate the base points of the ideal $J_{p}$ (see [8, 7.2]) coincide with those in $\mathcal{C}_{\mathcal{F}, p}$. As a consequence, $\mathcal{C}_{\mathcal{F}, p}$ is totally ordered by the relation $\geq$.

- $\mathcal{F}$ is not linearizable at $p$. Then there exist local analytic coordinates $u, v$ providing the Poincaré-Dulac normal form of $\mathcal{F}_{p}$, which is $\left(n u+v^{n}\right) d u-v d v$ for a certain positive integer $n$. Using this fact, it is straightforward to see that, as in the previous case, the set $\mathcal{C}_{\mathcal{F}, p}$ is totally ordered by the relation $\geq$. Observe that this case does not occur when $\mathcal{F}$ admits a rational first integral.

Each set $\mathcal{C}_{\mathcal{F}, p}$ defines, then, a valuation of the fraction field of $\mathcal{O}_{\mathbb{P}^{2}, p}$ and a simple complete primary ideal $I_{p}$ of that local ring [26, pages 389 to 391]; in fact, when $\mathcal{F}$ is linearizable at $p, I_{p} \mathcal{O}_{\mathbb{P}^{2}, p}^{a n}$ coincides with the integral closure of $J_{p}$ in $\mathcal{O}_{\mathbb{P}^{2}, p}^{a n}$. Set $D_{p}(\mathcal{F})$ to be the unique exceptionally supported divisor on $X_{\mathcal{F}}$ such that $I_{p} \mathcal{O}_{X_{\mathcal{F}}}=$ $\mathcal{O}_{X_{\mathcal{F}}}\left(-D_{p}(\mathcal{F})\right)$ and, for each positive integer $d$ and each map $\mathbf{k}: N \operatorname{Red}(\mathcal{F}) \rightarrow \mathbb{Z}_{+}$, denote by $\mathcal{L}(\mathcal{F}, d, \mathbf{k})$ the sheaf $\pi_{\mathcal{F}^{*}} \mathcal{O}_{\mathbb{P}^{2}}(d) \otimes \mathcal{O}_{X_{\mathcal{F}}}\left(-\sum \mathbf{k}(p) D_{p}(\mathcal{F})\right)$, where the sum is taken over the set of non-reduced singularities of $\mathcal{F}$.

We shall use the above notation in the statement and proof of the next result, which characterizes those non-degenerate plane foliations of degree $r \neq 1$ admitting a rational first integral. 
Theorem 3.7. Let $\mathcal{F}$ be a non-degenerate foliation on $\mathbb{P}^{2}$ of degree $r \neq 1$ and let $\left\{\left(\delta_{p}, \rho_{p}\right)\right\}_{p \in N \operatorname{Red}(\mathcal{F})}$ be the set of pairs of eigenvalues associated with the nonreduced singularities. Then, $\mathcal{F}$ has a rational first integral if and only if there exist a positive integer $d$ and a map $\mathbf{k}: N \operatorname{Red}(\mathcal{F}) \rightarrow \mathbb{Z}_{+}$such that:

(a) $d^{2}=\sum_{p \in N \operatorname{Red}(\mathcal{F})} \mathbf{k}(p)^{2} \rho_{p} \delta_{p}$.

(b) $d(r+2)=\sum_{p \in N \operatorname{Red}(\mathcal{F})} \mathbf{k}(p)\left(\rho_{p}+\delta_{p}\right)$.

(c) $h^{0}\left(X_{\mathcal{F}}, \mathcal{L}(\mathcal{F}, d, \mathbf{k})\right)=2$.

(d) There exist two curves of $\pi_{\mathcal{F} *}|\mathcal{L}(\mathcal{F}, d, \mathbf{k})|$ (say, with equations $H_{1}=0$ and $H_{2}=0$ ) such that, for each $p \in N \operatorname{Red}(\mathcal{F})$, the germs at $p$ of the curves with equations $H_{1}=0$ and $H_{2}=0$ are equisingular and the one of $H_{1} H_{2}=0$ satisfies the following conditions: it is reduced, its associated Milnor and Tjurina numbers coincide and its topological singularity type is $S\left(\rho_{p}, \delta_{p}, 2 \mathbf{k}(p)\right)$.

(e) Each point in $\operatorname{Red}(\mathcal{F})$ is a singular point of some curve in $\pi_{\mathcal{F}_{*}}|\mathcal{L}(\mathcal{F}, d, \mathbf{k})|$.

Moreover, in this case, a primitive rational first integral of $\mathcal{F}$ is $F / G$, where $F$ and $G$ are two homogeneous polynomials defining any two different curves in $\pi_{\mathcal{F} *}|\mathcal{L}(\mathcal{F}, d, \mathbf{k})|$.

Proof. Assume first that $\mathcal{F}$ has a rational first integral and let $R=F / G$ be a primitive one. Let $d$ denote the degree of $F$ and $G$. The morphism $\pi_{\mathcal{F}}$ defined above is the one eliminating the base points of the pencil $\mathcal{P}(R)$ or, equivalently, eliminating the indeterminacies of the rational map $\mathbb{P}^{2} \cdots \rightarrow \mathbb{P}^{1}$ provided by $R$ [2, II.7]. Moreover, for each $p \in N \operatorname{Red}(\mathcal{F})$, if $k_{p}$ denotes the number of branches through $p$ of a general curve $C$ of the pencil $\mathcal{P}(R)$, the multiplicities $m_{q}$ of the strict transforms of $C$ at the points $q \in \mathcal{C}_{\mathcal{F}, p}$ coincide with the ones of the strict transforms of any general element of the ideal $I_{p}^{k_{p}}$ (since $I_{p} \mathcal{O}_{\mathbb{P}^{2}, p}^{a n}$ is the integral closure of $\left.J_{p}\right)$. Thus, taking the function $\mathbf{k}: N \operatorname{Red}(\mathcal{F}) \rightarrow \mathbb{Z}_{+}$such that $\mathbf{k}(p)=$ $k_{p}$ for all $p \in N \operatorname{Red}(\mathcal{F})$, the following equality of sheaves holds: $\mathcal{L}(\mathcal{F}, d, \mathbf{k})=$ $\pi_{\mathcal{F}}^{*} \mathcal{O}_{\mathbb{P}^{2}}(d) \otimes \mathcal{O}_{X_{\mathcal{F}}}\left(\sum m_{q} E_{q}^{*}\right)$, where the sum is taken over the points $q \in \mathcal{C}_{\mathcal{F}}$ and $E_{q}^{*}$ denotes the pull-back on $X_{\mathcal{F}}$ of the exceptional divisor appearing in the blow-up centered at $q$. Since $\mathcal{P}(R)$ coincides with the pencil $\mathcal{P}_{\mathcal{F}}$ given in [12, Lem. 1], we can apply this result to deduce that $\mathcal{P}(R)=\pi_{\mathcal{F}_{*}}|\mathcal{L}(\mathcal{F}, d, \mathbf{k})|$; hence (c) is satisfied. Clauses (d) and (e) follow from Proposition [3.6. (a) is a consequence of applying Bézout's Theorem to two general curves in $\mathcal{P}(R)$ and (b) follows from a result in 21.

Conversely, assume the existence of a positive integer $d$, a map $\mathbf{k}: N \operatorname{Red}(\mathcal{F}) \rightarrow$ $\mathbb{Z}_{+}$and curves $H_{1}=0$ and $H_{2}=0$ as in the statement. Set $R=H_{1} / H_{2}$ and let $\mathcal{H}$ be the foliation provided by the derivation of the rational function $R$. Using Condition (d) and similar arguments to those given in the last part of the proof of Proposition 3.6. one can deduce that, for each $p \in N \operatorname{Red}(\mathcal{F})$, the Milnor number $\mu_{p}(\mathcal{H})$ equals 1 , the germs at $p$ of $H_{1}=0$ and $H_{2}=0$ have singularity type $S\left(\rho_{p}, \delta_{p}, \mathbf{k}(p)\right)$ and, moreover, they have the same minimal resolution of singularities. From the last assertion one has also that $i_{p}\left(H_{1}=0, H_{2}=0\right)=\mathbf{k}(p)^{2} \rho_{p} \delta_{p}$, where $i_{p}$ stands for the intersection multiplicity at $p$. This fact, Bézout's Theorem and Condition (a) show that the base points on $\mathbb{P}^{2}$ of the pencil $\mathcal{P}(R)=\pi_{\mathcal{F} *}|\mathcal{L}(\mathcal{F}, d, \mathbf{k})|$ (the equality holds from $(\mathrm{c}))$ are exactly those in $N \operatorname{Red}(\mathcal{F})$. Hence, $N \operatorname{Red}(\mathcal{H})=N \operatorname{Red}(\mathcal{F})$.

It follows from the above paragraph that the pair of eigenvalues attached to each non-reduced singularity $p$ of $\mathcal{H}$ is $\left(\delta_{p}, \rho_{p}\right)$ and the number of branches through $p$ 
of a general curve of the pencil $\mathcal{P}(R)$ is $\mathbf{k}(p)$. Then, if $r^{\prime}$ denotes the degree of the foliation $\mathcal{H}$, the equality $d\left(r^{\prime}+2\right)=\sum_{p \in N \operatorname{Red}(\mathcal{F})} \mathbf{k}(p)\left(\rho_{p}+\delta_{p}\right)$ holds [21]. By Condition (b), we deduce that $r=r^{\prime}$. Therefore,

$$
\sum_{p \in \operatorname{Sing}(\mathcal{H})} \mu_{p}(\mathcal{H})=r^{2}+r+1
$$

and so, \#Red $(\mathcal{H}) \leq \# \operatorname{Red}(\mathcal{F})$, where \# stands for the cardinality. But, by Condition (e) and Part (b) of Lemma 3.5, each point in $\operatorname{Red}(\mathcal{F})$ is also a singular point of $\mathcal{H}$. Hence one gets that $\operatorname{Ind}(R)=N \operatorname{Red}(\mathcal{H})=N \operatorname{Red}(\mathcal{F}), \operatorname{Sing}(R)=\operatorname{Red}(\mathcal{H})=$ $\operatorname{Red}(\mathcal{F})$ and, taking into account (3.1), $\mathcal{H}$ is a non-degenerate foliation. Thus, by Proposition 3.6, $R$ is a rational first integral of $\mathcal{F}$.

Finally, since $h^{0}(X, \mathcal{L}(\mathcal{F}, d, \mathbf{k}))=2$ we deduce that $R$ is a primitive rational first integral of $\mathcal{F}$ by the proof of [12, Th. 2 (a)].

Remark 3.8. Note that, if Condition (d) in the statement of the above result is satisfied for two curves of $\pi_{\mathcal{F}_{*}}|\mathcal{L}(\mathcal{F}, d, \mathbf{k})|$, then it holds for any pair of general elements of this linear system.

Example 3.9. The pair of Diophantine equations of a fixed non-degenerate foliation $\mathcal{F}$ given by clauses (a) and (b) of Theorem 3.7 where the unknowns are $d$ and $\{\mathbf{k}(p)\}_{p \in N \operatorname{Red}(\mathcal{F})}$, can have infinitely many solutions. Indeed, foliations in the set $\left\{\mathcal{F}_{\alpha}^{r}\right\}_{\alpha \in E}, 2 \leq r \leq 4$, given in [17. ( $E$ is a countable and dense set of parameters in the set of complex numbers), have singularities of fixed analytic type 17, Def. 1], rational first integral and their degrees can be chosen arbitrarily large. Therefore we get the Diophantine equations

$$
\begin{array}{c||c||c}
d^{2}=2 \sum_{i=1}^{2} k_{i}^{2}+6 \sum_{i=3}^{5} k_{i}^{2} & d^{2}=\sum_{i=1}^{3} k_{i}^{2}+2 \sum_{i=4}^{8} k_{i}^{2} & d^{2}=\sum_{i=1}^{12} k_{i}^{2} \\
4 d=3 \sum_{i=1}^{2} k_{i}+5 \sum_{i=3}^{5} k_{i} & 5 d=2 \sum_{i=1}^{3} k_{i}+3 \sum_{i=4}^{8} k_{i} & 3 d=\sum_{i=1}^{12} k_{i}
\end{array}
$$

associated, respectively, to the cases $r=2,3,4$, where for simplicity we have set $\mathbf{k}\left(p_{i}\right)=k_{i}$, which have infinitely many solutions (attached to the corresponding first integrals).

To end this example, we examine clauses in Theorem 3.7 for the foliation $\mathcal{F}_{0}^{4}$ that is given by the 1-form $A d X+B d Y+C d Z$, where $A=\left(Y^{3}-Z^{3}\right) Y Z, B=$ $\left(Z^{3}-X^{3}\right) X Z$ and $C=\left(X^{3}-Y^{3}\right) X Y$. It has 12 points in $N R e d(\mathcal{F})$ and 9 points in $\operatorname{Red}(\mathcal{F})$. As $\mathcal{F}_{0}^{4}$ has rational first integral, clauses (a) to (e) in Theorem 3.7 must hold. In fact, $d=6, k_{1}=k_{2}=k_{3}=3$ and $k_{i}=1,4 \leq i \leq 12$ are solutions for the above last Diophantine equations. Forms $H_{1}=3 X^{3} Y^{3}-X^{3} Z^{3}-2 Y^{3} Z^{3}$ and $H_{2}=2 X^{3} Y^{3}-X^{3} Z^{3}-Y^{3} Z^{3}$ span the vector space $H^{0}\left(X_{\mathcal{F}}, \mathcal{L}(\mathcal{F}, 6, \mathbf{k})\right)$ for $\mathbf{k}$ defined as above and a suitable ordering of the points in $N \operatorname{Red}(\mathcal{F})$. The equation of the germ of the curve defined by $H_{1}\left(H_{2}\right.$, respectively) at the point $p=(0: 0$ : $1) \in N \operatorname{Red}(\mathcal{F})$ (whose image by $\mathbf{k}$ equals 3 ) in suitable local coordinates is given by $h_{1}=x^{3}+2 y^{3}-3 x^{3} y^{3}\left(h_{2}=x^{3}+y^{3}-2 x^{3} y^{3}\right.$, respectively). The germ defined by $h_{1} h_{2}$ factorizes into a product of 6 smooth transversal analytically irreducible germs passing through $p$ (having, thus, singularity type $S(1,1,6)$ ) and its Milnor and Tjurina numbers are both equal to 25 (we have used Singular 14 to do the computation); obviously the germs defined by $h_{1}$ and $h_{2}$ are equisingular. The same situation happens for the two remaining points of $N \operatorname{Red}(\mathcal{F})$ whose image by $\mathbf{k}$ is 3 . The germs of $H_{1}$ and $H_{2}$ at any point $q \in N \operatorname{Red}(\mathcal{F})$ such that $\mathbf{k}(q)=1$ are analytically irreducible, smooth and transversal. This shows that the conditions given in (d) are satisfied for $H_{1}$ and $H_{2}$. Finally (e) also holds because the points 
$\left(j^{r}: 0: 1\right)\left(\left(1: j^{r}: 0\right)\right.$, respectively) $\left(\left(0: j^{r}: 1\right)\right.$, respectively), where $r \in\{0,1,2\}$ and $j:=e^{2 \pi i / 3}$, are singular points of $Y^{3}\left(X^{3}-Z^{3}\right)\left(Z^{3}\left(Y^{3}-X^{3}\right)\right.$, respectively $)$ $\left(X^{3}\left(Y^{3}-Z^{3}\right)\right.$, respectively).

Remark 3.10. The unique non-degenerate foliations of degree one are defined, up to projective isomorphism, by the differential 1-forms $a Y Z d X+b X Z d Y-(a+$ b) $X Y d Z$, where $a$ and $b$ are positive integers, $(X: Y: Z)$ being projective coordinates on the complex projective plane (see [6, Sect. 4]). All of them are algebraically integrable, since $X^{a} Y^{b} / Z^{a+b}$ is a rational first integral.

Remark 3.11. Theorem 3.7 allows us to decide whether a non-degenerate foliation $\mathcal{F}$ of degree $r \neq 1$, defined by a projective differential 1-form $\Omega$, does or does not have a rational first integral $\frac{F}{G}$ such that the degree of $F$ and $G$ is less than a fixed value $t$ (and to compute it, if it exists). The procedure should be as follows: firstly, perform a minimal resolution of $\mathcal{F}$ and compute the divisors $D_{p}(\mathcal{F})$ for all $p \in N \operatorname{Red}(\mathcal{F})$. Secondly, consider the integers $d<t$ and the finite set of maps $\mathbf{k}: N \operatorname{Red}(\mathcal{F}) \rightarrow \mathbb{Z}_{+}$ satisfying the conditions (a), (b) and (c) of Theorem 3.7. Then, $\mathcal{F}$ has a rational first integral with the above condition if and only if $d\left(\frac{F}{G}\right) \wedge \Omega=0$ for some basis $\{F, G\}$ of a linear system of the type $\pi_{\mathcal{F} *}|\mathcal{L}(\mathcal{F}, d, \mathbf{k})|$.

Example 3.12. Take projective coordinates $(X: Y: Z)$ on the complex projective plane and consider the non-degenerate foliation $\mathcal{F}$ defined by the projective differential 1-form $\Omega=A d X+B d Y+C d Z$, where

$$
A=Z\left(2 X^{3}+2 Y^{3}-Y Z^{2}\right), \quad B=Z\left(X Z^{2}-6 X Y^{2}\right) \text { and } C=4 X Y^{3}-2 X^{4} \text {. }
$$

It has 6 non-reduced singularities $p_{1}, p_{2}, \ldots, p_{6}$ and the morphism $\pi_{\mathcal{F}}: X_{\mathcal{F}} \rightarrow \mathbb{P}^{2}$ is the composition of the blow-ups with centers $p_{1}, p_{1}^{\prime}, p_{2}, p_{2}^{\prime}, p_{3}, p_{3}^{\prime}, p_{4}, p_{5}$ and $p_{6}$, where $p_{i}^{\prime}$ is infinitely near to $p_{i}$ for $1 \leq i \leq 3$. The pair of eigenvalues associated with $p_{i}, 1 \leq i \leq 3$ ( $4 \leq i \leq 6$, respectively), is $(1,2)((1,1)$, respectively). Using results of [18] we see that the divisor $D_{p_{i}}(\mathcal{F})$ is $E_{p_{i}}^{*}+E_{p_{i}^{\prime}}^{*}\left(E_{p_{i}}^{*}\right.$, respectively) for $1 \leq i \leq 3$ ( $4 \leq i \leq 6$, respectively), where $E_{q}^{*}$ denotes the total transform on $X_{\mathcal{F}}$ of the exceptional divisor appearing in the blow-up centered at $q$. The integer $d=3$ and the map $\mathbf{k} \equiv 1$ satisfy the conditions (a), (b) and (c) of Theorem 3.7 and the linear system $\pi_{\mathcal{F} *}|\mathcal{L}(\mathcal{F}, 3, \mathbf{k})|$ is spanned by the curves with equations $F:=X^{3}-2 Y^{3}+Y Z^{2}=0$ and $G:=X Z^{2}=0$. After checking it, we assert that $F / G$ is a rational first integral of $\mathcal{F}$.

When a non-degenerate plane foliation $\mathcal{F}$ has a rational first integral, the cardinality of the set $N \operatorname{Red}(\mathcal{F})$ of non-reduced singularities is not arbitrary, as the next result shows. Notice that this gives an easy to check criterion to decide that a foliation has no rational first integral.

Theorem 3.13. Let $\mathcal{F}$ be a degree $r$ non-degenerate foliation on $\mathbb{P}^{2}$ which has a rational first integral. Then

$$
r+1 \leq n,
$$

where $n$ is the number of non-reduced singularities of $\mathcal{F}$.

Proof. Let $\left\{\left(\delta_{p}, \rho_{p}\right)\right\}_{p \in N \operatorname{Red}(\mathcal{F})}$ be the set of pairs of eigenvalues associated with the non-reduced singularities of the foliation $\mathcal{F}$. Consider a primitive rational first integral of $\mathcal{F}, R$, and let $C$ be a general curve of the pencil $\mathcal{P}(R)$. For each $p \in N \operatorname{Red}(\mathcal{F})$, let $k_{p}$ be the number of branches of the singularity of $C$ at $p$. Assume that $C$ has degree $d$. Bertini's Theorem proves that the unique singularities of $C$ are 
the non-reduced singularities of $\mathcal{F}$ and similar arguments to some used in the proof of Proposition 3.6 show that each non-reduced singularity $p$ is quasi-homogeneous with associated weights $\left(k_{p} \rho_{p}, k_{p} \delta_{p}\right)$. So, in suitable coordinates $\{x, y\}$ in $\mathcal{O}_{\mathbb{P}^{2}, p}^{a n}$, the germ of $C$ at $p$ is given by $H\left(x^{\rho_{p}}, y^{\delta_{p}}\right), H$ being a homogeneous polynomial in two indeterminates of degree $k_{p}$. So the Milnor and Tjurina numbers coincide at each singularity of $C$.

Let $\mu$ denote the sum of Milnor numbers of the singularities of $C$. Then,

$$
(d-1)(d-r-1) \leq \mu
$$

by a result of du Plessis and Wall [10. $C$ is a general curve of the pencil $\mathcal{P}(R)$ and, so, the Milnor-Jung formula states that

$$
\mu_{p}=2 \bar{\delta}_{p}-k_{p}+1
$$

for all $p \in N \operatorname{Red}(\mathcal{F})$, where $\mu_{p}=\mu(C, p)\left(\bar{\delta}_{p}\right.$, respectively) is the Milnor number (genus diminution or $\delta$-invariant, respectively) of $C$ at the singularity $p$. Hence,

$$
\mu=\sum_{p \in N \operatorname{Red}(\mathcal{F})}\left(2 \bar{\delta}_{p}-k_{p}\right)+n .
$$

On the one hand, by the genus formulae, one gets:

$$
2 g-2=C \cdot C+C \cdot K_{\mathbb{P}^{2}}-\sum_{p \in N \operatorname{Red}(\mathcal{F})} k_{p}^{2} \rho_{p} \delta_{p}+\sum_{p \in N \operatorname{Red}(\mathcal{F})} k_{p}\left(\rho_{p}+\delta_{p}-1\right),
$$

where $g$ is the geometrical genus of $C$ and $K_{\mathbb{P}^{2}}$ a canonical divisor of the projective plane (see [15, pages 279 and 280]). On the other hand, it follows that

$$
2 g=(d-1)(d-2)-2 \sum_{p \in N \operatorname{Red}(\mathcal{F})} \bar{\delta}_{p}
$$

(see, for instance, [16, Sect. IV, Ex. 1.8]). Then, we obtain the following equality:

$$
\mu=\sum_{p \in N \operatorname{Red}(\mathcal{F})}\left[k_{p}^{2} \rho_{p} \delta_{p}-k_{p}\left(\rho_{p}+\delta_{p}\right)\right]+n
$$

Finally,

$$
\mu=d^{2}-(r+2) d+n,
$$

since $d^{2}=\sum_{p \in N \operatorname{Red}(\mathcal{F})} k_{p}^{2} \rho_{p} \delta_{p}$ and $\sum_{p \in N \operatorname{Red}(\mathcal{F})} k_{p}\left(\rho_{p}+\delta_{p}\right)=(r+2) d$ [21], which concludes the proof after replacing in (3.2) the value of $\mu$.

Example 3.14. For each $a \in \mathbb{C} \backslash\{0,1,-1\}$, consider the non-degenerate plane foliation $\mathcal{F}_{a}$ defined by the projective 1 -form $\Omega=A d X+B d Y+C d Z,(X: Y: Z)$ being projective coordinates in the complex projective plane, where:

$$
\begin{gathered}
A=Z\left(a X Z-Y^{2}+Z^{2}\right), \quad B=Z\left(X^{2}-Z^{2}\right) \text { and } \\
C=X Y^{2}-a X^{2} Z-X Z^{2}-X^{2} Y+Y Z^{2} .
\end{gathered}
$$

The cardinality of $N \operatorname{Red}\left(\mathcal{F}_{a}\right)$ is 1 or 2 , depending on the value of the parameter $a$ (see [12, Example 5]). Since the degree of the foliation is 2, Theorem 3.13 allows us to discard the existence of a rational first integral for any foliation in the family $\left\{\mathcal{F}_{a}\right\}_{a \in \mathbb{C} \backslash\{0,1,-1\}}$.

Finally, we give an example which shows that the lower bound of Theorem 3.13 turns out to be sharp. 
Example 3.15. Consider any foliation $\mathcal{F}$ of the family given in Remark $3.10, \mathcal{F}$ has degree 1 and it has three singular points: $(0: 0: 1),(0: 1: 0)$ and $(1: 0: 0)$. A rational first integral of $\mathcal{F}$ is $\frac{X^{a} Y^{b}}{Z^{a+b}}$ (for suitable $a$ and $b$ ) and, so, the non-reduced singularities are $(0: 1: 0)$ and $(1: 0: 0)$.

\section{REFERENCES}

1. L. Autonne, Sur la théorie des équations différentielles du premier ordre et du premier degré, J. École Polytech. 61 (1891), 35-122; 62 (1892), 47-180.

2. A. Beauville, Complex algebraic surfaces, London Math. Soc. Student Texts 34, Cambridge University Press, 1996. MR.1406314 (97e:14045)

3. M. Brunella, Birational Geometry of Foliations, Springer-Verlag, 2000. MR.1948251 (2004g:14018)

4. C. Camacho and P. Sad, Pontos singulares de equações diferenciais analíticas, 16 Colóq. Bras. Mat., IMPA, 1987. MR953780 (90a:58126)

5. A. Campillo and M. Carnicer, Proximity inequalities and bounds for the degree of invariant curves by foliations of $\mathbb{P}_{\mathbb{C}}^{2}$, Trans. Amer. Math. Soc. 349 (9) (1997), 2211-2228. MR1407696 (97h:32051)

6. A. Campillo and J. Olivares, Polarity with respect to a foliation and Cayley-Bacharach Theorems, J. Reine Angew. Math. 534 (2001), 95-118. MR1831632 (2002c:32051)

7. M. Carnicer, The Poincaré problem in the nondicritical case, Ann. of Math. (2) 140 (1994), 289-294. MR,1298714 (95k:32031)

8. E. Casas-Alvero, Singularities of plane curves, London Math. Soc. Lecture Notes 276, Cambridge University Press, 2000. MR:1782072 (2003b:14035)

9. G. Darboux, Mémoire sur les équations différentielles algébriques du premier ordre et du premier degré (Mélanges), Bull. Sci. Math. 32 (1878), 60-96; 123-144; 151-200.

10. A. A. du Plessis and C. T. C. Wall, Application of the theory of the discriminant to highly singular plane curves, Math. Proc. Cambridge Phil. Soc. 126 (1999), 259-266. MR1670229 (99m:14053)

11. E. Esteves and S. Kleiman, Bounds on leaves of one-dimensional foliations, Bull. Braz. Math. Soc. (N.S.) 34(1) (2003), 145-169. MR.1993042 (2004m:32060)

12. C. Galindo and F. Monserrat, Algebraic integrability of foliations of the plane, J. Differential Equations 231 (2) (2006), 611-632. MR.2287899 (2008a:32028)

13. J. García de la Fuente, Geometría de los sistemas lineales de series de potencias en dos variables, Ph.D. thesis, Valladolid University, 1989.

14. G.M. Greuel, G. Pfister and H. Schöenemann, Singular 3.0. A Computer Algebra System for Polynomial Computations. Centre for Computer Algebra, University of Kaiserslautern, 2005. http://www. singular.uni-kl.de.

15. P. Griffiths and J. Harris, Principles of algebraic geometry, Wiley Interscience, 1978. MR.507725 (80b:14001)

16. R. Hartshorne, Algebraic geometry, Springer-Verlag, 1977. MR0463157 (57:3116)

17. A. Lins-Neto, Some examples for the Poincaré and Painlevé problems, Ann. Sc. École Norm. Sup. (4) 35 (2002), 231-266. MR1914932 (2003j:34009)

18. J. Lipman, Proximity inequalities for complete ideals in two-dimensional regular local rings, Contemp. Math. 159 (1994), 293-306. MR.1266187 (95j:13018)

19. P. Painlevé, Sur les intégrales algébriques des équations différentielles du premier ordre and Mémoire sur les équations différentielles du premier ordre in Oeuvres de Paul Painlevé, Tome II, Éditions du Centre National de la Recherche Scientifique 15, quai Anatole-France, Paris 1974.

20. H. Poincaré, Mémoire sur les courbes définies par les équations différentielles, J. Math. Pures Appl. 3 (7) (1881), 375-442; 3 (8) (1882), 251-296; 4 (1) (1885), 167-244; in Oeuvres de Henri Poincaré, vol. I, Gauthier-Villars, Paris, 1951, 3-84, 95-114.

21. Sur l'intégration algébrique des équations différentielles du premier ordre et du premier degré (I), Rend. Circ. Mat. Palermo 5 (1891), 161-191.

22. __ Sur l'intégration algébrique des équations différentielles du premier ordre et du premier degré (II), Rend. Circ. Mat. Palermo 11 (1897), 193-239. 
23. K. Saito, Quasihomogene isolierte singularitäten von hyperflächen, Invent. Math. 14 (1971), 123-142. MR0294699 (45:3767)

24. A. Seidenberg, Reduction of singularities of the differential equation Ady $=B d x$, Amer. J. Math. 90 (1968), 248-269. MR0220710 (36:3762)

25. E. Yoshinaga and M. Suzuki, Topological types of quasihomogeneous singularities in $\mathbb{C}^{2}$, Topology 18 (1979), 113-116. MR544152 (80k:32017)

26. O. Zariski and P. Samuel, Commutative algebra, Vol. II, Springer-Verlag, 1960. MR0120249 $(22: 11006)$

Departamento de Matemáticas and Instituto Universitario de Matemáticas y ApliCaciones de Castellón (IMAC), Universidad Jaume I, Campus Riu Sec, 12071 Castellón, SPAIN

E-mail address: galindo@mat.uji.es

Instituto Universitario de Matemática Pura y Aplicada, Universidad Politécnica de Valencia, Camino de Vera s/n, 46022 Valencia, Spain

E-mail address: framonde@mat.upv.es 